\title{
»Buchbesprechungen $«$
}

Wolfgang Däubler/Gudrun Küsel (Hg.), Verfassungsgericht und Politik. Kritische Beiträge zu problematischen Urteilen, Reinbek $b$. Hamburg 1979 (Rowoblt Taschenbuch Verlag), 223 S., $D M 7,80$

Friedhelm Hase / Karl-Heinz Ladeur, Verfassungsgerichtsbarkeit und politisches System: Studien zum Rechtsstaatsproblem in Deutschland; Frankfurt/M., New York 1980 (Campus Verlag), 33I, S., DM 38,-

Reinhold Schlothauer, Zur Krise der Verfassungsgerichtsbarkeit - Neuere Ansätze zur Methodik der Verfassungsinterpretation: unters. am Beispiel von Horst Ehmke, Peter Häberle, Konrad Hesse, Martin Kriele u. Friedrich Müller; Köln, Frankfurt/M. 1979 (Europäische Verlagsanstalt), 224 S., $\mathrm{DM}_{3} 8$,-

I.

Für einen »Hüter « ist das Bundesverfassungsgericht von der kritischen Rechtstheorie und von der sozialwissenschaftlich angeleiteten empirischen Forschung bislang im Vergleich zu anderen politischen Institutionen eher stiefmütterlich behandelt worden. Konservative und auch in der liberalen Tradition stehende Staatsrechtler betrachteten Institution und Spruchpraxis des BVerfG vorwiegend aus strikt normwissenschaftlicher Sicht. Die theoretisch-praktische Auseinandersetzung der Linken mit dem BVerfG hatte vor allem in den fünfziger und sechziger Jahren in erster Linie eine durch den » Kampf um Verfassungspositionen « diktierte historisch notwendige, aber dennoch eingeengte strategische Perspektive.'

Auch die Renaissance marxistischer Theorie-

I J. Seifert, Kampf um Verfassungspositionen, Frankfurt/M. 1974; W. Abendroth et al., Diskussion über Probleme sozialistischer Rechtspolitik, in H. Rottleuthner (Hg.), Probleme der marxistischen Rechtstheorie, Frankfurt/M. 1975, S. $392 \mathrm{ff}$ bildung Ende der 6oer und Anfang der 7oer Jahre ließ Institution und Funktion der Verfassungsgerichtsbarkeit weitgehend unberührt. ${ }^{2}$ Dies mag auf deren kurze bundesrepublikanische Wirkungsgeschichte zurückzuführen sein oder auf ihre damals nachrangige theoretische Bedeutung als »Überbauphänomen «. Jedenfalls ist festzustellen, daß die marxistisch inspirierte Verfassungstheorie bemerkenswert »bürgerlich« informiert blieb. Sie übernahm die Befunde und unter der Hand auch die Fragestellung liberaler, bisweilen auch konservativer, normwissenschaftlich begrenzter Untersuchungen und kritisierte (wie diese) vorwiegend Kompetenzüberschreitungen, mangelnden sself-restraint<, Verzerrungen der funktionell-rechtlichen Balance zwischen Gesetzgeber und Gericht, das hypertrophe Selbstverständnis und die unzureichende formaldemokratische Legitimation des BVerfG. ${ }^{3}$ Freilich ist das Defizit an systematischer empirischer Forschung ${ }^{4}$, die sich im Bezugsrahmen einer marxistischen Gesellschaftstheorie bewegt, nicht primär den Forschern, sondern eher der politischen Borniertheit und den vor allem finanziellen Restriktionen des Wissenschaftssystems anzulasten. Dennoch bleibt es ein bedauerlicher Mangel, der die Frage nahelegt, ob und inwieweit die hier rezensierten Studien zur Verfassungsgerichtsbarkeit diesem Mangel abhelfen.

2 Vgl. dazu T. Blanke, Das Dilemma der verfassungspolitischen Diskussion der Linken in der Bundesrepublik, in: H. Rottleuthner, a. a. O., S. $419 \mathrm{ff}$.

3 Vgl. die Zusammenfassung der Diskussion bei E. Denninger, Staatsrecht 2, Reinbek b. Hamburg 1979, Kap. V, m. zahlr. Nachw.

4 Zur empirischen Forschung und zu den theoretischen Arbeiten über Institution, Geschichte und Funktion der Verfassungsgerichtsbarkeit vgl. Josef Mackert, Bibliographie zur Verfassungsgerichtsbarkeit des Bundes und der Länder, I und II, Frankfurt/M. 1971/1976. 
II.

Die von Däubler/Küsel herausgegebenen »Kritischen Beiträge zu problematischen Urteilen « weisen bereits im Untertitel den Anspruch einer systematischen Analyse der Institution und Spruchpraxis des BVerfG zurück. Wie im Vorwort unprätentiös und allgemein formuliert, lassen sich die Autoren der zwölf Beiträge von der Absicht leiten, die »Desinformiertheit der Öffentlichkeit über die verfassungsrechtliche Problematik abzubauen «, also die Diskussion nicht (mehr) nur zunftintern zu führen. Daß eine solche »Veröffentlichung « des juristischen Diskurses notwendig ist, bedarf keiner näheren Erläuterung. Ist sie den Autoren gelungen? Eine erste Voraussetzung - scheinbar trivial, aber gerade von den Akteuren im wissenschaftlichen Reputationssystem nicht leicht zu erfüllen - ist die Sprache der Darstellung und Kritik. Den Autoren kann bescheinigt werden, daß sie Beiträge vorgelegt haben, die lesbar sind und sich dem Zugriff der "praktischen Vernunft « interessierter Nichtjuristen erschließen müßten.

Auch die Auswahl der Beiträge beweist die Ernsthaftigkeit des »Veröffentlichungs «-Interesses: Von unterschiedlichen methodisch-theoretischen Ansatzpunkten und politischen Standorten und mit je unterschiedlichem Erkenntnisinteresse untersuchen die Autoren (a) einzelne Entscheidungen des BVerfG (Mitbestimmungsurteil und Entscheidung zum Brem. Personalvertretungsgesetz / U. Mückenberger), (b) die Judikatur zu bestimmten Problemkomplexen (Wirtschaftsverfassung des GG / N. Reich; Berufsbeamtentum / Chr. Müller; Meinungsfreiheit / K. H. Ladeur; "Außenbeziehungen « der BRD / W. Däubler; Ausländerrecht / H. Rittstieg), (c) Aspekte der Wirkungsgeschichte des BVerfG (Bedeutung für die Konstituierung der Demokratie / F. Dopatka; Selbstverständnis / T. Rasehorn; praktische Umsetzung verfassungsgerichtlicher Entscheidungen / H. Wagner; Zukunftsperspektiven / K. Zweigert und H. Dietrich; Vergleich zur Verfassungsgerichtsbarkeit anderer Staaten / M. Hirsch; Demokratieangemessene Rolle des BVerfG / W. Holtfort). Den Autoren ist zugutezuhalten, daß sie nicht akademische Steckenpferde in die realitätsverdünnte Luft esoterischer Problemzonen geritten haben, sondern in informationsreichen Beiträgen die Existenz und Praxis der Verfassungsgerichtsbarkeit diskutieren.
Die empirischen Befunde, die in den einzelnen Beiträgen zur Spruchpraxis und Funktion des BVerfG erhoben werden, decken sich weitgehend: Uminterpretation der Verfassung in ein geschlossenes Normprogramm, Verrechtlichung der Politik, Usurpation gesetzgeberischer Aufgaben. Auch besteht Übereinstimmung dahingehend, daß die genuine Aufgabe eines Verfassungsgerichts der Grundrechtsschutz sein müßte. Kritik und Reformvorschläge orientieren sich überwiegend an den Bedingungen und Prinzipien repräsentativ-parlamentarischer Demokratie.

Institution, Selbstverständnis und Spruchpraxis des BVerfG werden von Zweigert/Dietrich im Hinblick auf die Zukunftsaussichten der Verfassungsgerichtsbarkeit in der Bundesrepublik untersucht. Ausgangspunkt ihrer Überlegungen ist die These eines faktischen Legitimationsdefizits des BVerfG (vgl. dazu den Beitrag von Rasehorn):

»(E)ine Verfassungsgerichtsbarkeit derzeitigen Zuschnitts findet heute kaum mehr die nötige Zustimmung der mündigen Bevölkerung.« (I I f.) Es fragt sich jedoch, ob dieses Defizit mit der »Verschiebung des institutionellen Gefüges der checks und balances hin zum Verfassungsgericht« (I 2) zureichend erklärt. Zweigert/Dietrich analysieren exemplarisch einige Entscheidungen des BVerfG Grundlagenvertrag, Diätenurteil, \218, Wehrdienstnovelle - und kritisieren diese pausibel unter dem Gesichtspunkt der Arbeits- und Kompetenzverteilung innerhalb des politischen Systems. Im Verlauf der letzten Jahre haben sich die politischen Gewichte institutionell gewiß zugunsten des Verfassungsgerichts verschoben. Unbestreitbar ist auch, daß die Verrechtlichung - genauer wohl: die verfassungsgerichtliche Justizialisierung - des politischen Entscheidungsprozesses "antidemokratische Effekte« gehabt hat, wie sie Zweigert/Dietrich charakterisieren: "Insgesamt legt sich damit ein immer dichter geknüpftes Netz von juristischen Normen extremen Härtegrads über den Handlungsspielraum der demokratisch bestimmten politischen Instanzen.

Damit sind tiefgreifende Konsequenzen für den demokratischen Prozeß verbunden. Auf der Ebene der Entscheidungsträger tritt eine Veränderung der Perspektive und damit eine Veränderung des Inhalts der politischen Entscheidungen ein. Politische Entscheidungen gehen nicht mehr in erster Linie von einer 
Erfassung des Sachproblems aus und stellen Lösungen aus der Gesamtheit der Tatsachen dar; die soziale Wirklichkeit wird vielmehr nur noch in den von den verfassungsrechtlichen Normen und Entscheidungen her vorgegebenen Kategorien erfaßt und gestaltet. Was, oft beklagt, aus Gerichtssälen her bekannt ist, daß nämlich die soziale Wirklichkeit nur soweit zur Kenntnis genommen wird, wie sie für die Normausfüllung relevant ist, wiederholt sich jetzt im Bereich der politischen Entscheidungen von Gesetzgeber und Regierung. An die Stelle einer Ermittlung des sozialen Sachverhalts und der Entwicklung innovativer Lösungen tritt rechtlich vorstrukturierte Wirklichkeitsperzeption und juristische Subsumtion. Die Perspektive der demokratischen Entscheidungsträger wird zunehmend zu einer juristischen. Die Konsequenzen dieser Entwicklung für die Gegenwart und zukunftige Gestaltung der Belange des Gemeinwesens können nur schrecken: der sich ständig verlängernde Schatten des verfassungsrechtlichen Normengefüges macht zunehmend eine offene Sozialgestaltung unmöglich.« (19)

Allerdings wären (a) die normative Legitimation und die faktische Legitimierbarkeit verfassungsgerichtlicher Entscheidungen genauer zu differenzieren und (b) die Institution Verfassungsgerichtsbarkeit einmal im gewaltenteiligen politischen System und zum anderen im Spannungsfeld der gesellschaftlichen Kräfte, der »intermediären Gewalten «, pressure groups, Minderheiten etc. zu verorten. Die Rechtsprechungskritik von Zweigert/Dietrich, die daraus entwickelten Reformvorschläge, die sich weitgehend mit denen von Holtfort (19I ff.) decken, überzeugen nur unter dem Gesichtspunkt der Gewaltenteilung: Mit richterlicher Selbstbeschränkung und der Einführung des Rechts, Entscheidungen $\mathrm{zu}$ verweigern (certiorari); mit der Begrenzung der Zuständigkeit und insbesondere der Abschaffung der abstrakten Normenkontrolle; mit der Beschränkung der verfassungsgerichtlichen Überprüfung auf konkrete Streitfälle (wie im amerikanischen System auf "cases and controversies") wäre für den Gesetzgeber Terrain zurückgewonnen. Mit einer "anderen Zusammensetzung des Richtergremiums«, so Rasehorns Aufforderung an die SPD, mit mehr Transparenz und Publizität (Hearings) bei der Richterwahl ließe sich u. U. erreichen, die Institution Verfassungsgerichtsbarkeit partiell demokra- tisch zu reformieren. Es ist in der Tat (normativ) »nicht einzusehen, warum eine parlamentarische Demokratie die Wahl in das mit höchster Autorität ausgestattete Verfassungsorgan nicht ebenso zum Gegenstand allgemeinen Interesses macht wie die Wahl etwa des Bundespräsidenten« (Holtfort, 195). Doch zeigt gerade das Beispiel des U.S. Supreme Court, auf dessen - wohl mehr Ideologie als Praxis des - self-restraint und auf dessen restringierte Kompetenzen sich viele Kritiker des BVerfG berufen, daß mit diesen Vorschlägen die prinzipiellen Einwände gegen die verfassungsgerichtliche Überprüfung von Parlamentsentscheidungen nicht ausgeräumt sind, und daß für die systematische Kritik an der Spruchpraxis noch kein leitender normativer Gesichtspunkt gefunden ist. (Im übrigen wäre hinsichtlich des self-restraint $\mathrm{zu}$ fragen, was darunter $\mathrm{zu}$ verstehen ist: Zurückhaltung beim Eigentumsschutz und Schutz politischer Freiheit? Oder nur Zurückhaltung bei der Überprüfung von staatlichen Maßnahmen, die die politischen Freiheitsrechte und den demokratischen Prozeß nicht tangieren?)

Wenn man, wie Holtfort, gegen ein "mit oberster Autorität ausgestattetes Richterkollegium«, das Mehrheitsentscheidungen kassieren kann, vornehmlich das der repräsentativen Demokratie zugrundeliegende Mehrheitsprinzip ins Feld führt und den Schutz von Minderheiten nur am Rande erwähnt, dann gibt es keine Gründe, die die Existenz und das Prüfungsrecht eines Verfassungsgerichts zwingend gebieten.

Andererseits ist dem Grundgesetz zu entnehmen, daß alle Staatsgewalt vom Volke ausgehen soll - womit die Staatsorganisation und der politische Willensbildungsprozeß auf das Fundamentalprinzip demokratischer Selbstregierung des Volkes festgelegt sind. Diese Staatsgewalt, so der zweite Schritt, »wird vom Volke in Wahlen und Abstimmungen ausgeübt«. Damit werden zwei Verfahren parlamentarische Wahl und Plebiszit - für die Äußerung des Volkswillens vorgesehen; das Prinzip der Volkssouveränität bleibt davon berührt. Wenn nun in einem dritten Schritt bestimmt wird, daß die Staatsgewalt »durch besondere Organe der Gesetzgebung, der vollziehenden Gewalt und der Rechtsprechung «, also gewaltenteilig ausgeübt werden soll, dann handelt es sich dabei um eine Ausübungsbefugnis, nicht aber um eine Verlagerung der Souveränität; der Absatz 3 des 
Art. 20 stellt das klar. Sinn dieser gewiß differenzierungsbedürftigen Argumentation "am Text« des Grundgesetzes ist, der Verkürzung des Prinzips der Selbstregierung auf das Mehrheitsprinzip und der impliziten Verdrängung der Volkssouveränität durch die Parlamentssouveränität entgegenzutreten eine Verkürzung, die nicht nur die Normativität des Grundgesetzes, insbesondere das Konstruktionsprinzip einer geschriebenen Verfassung, die politischen Freiheitsrechte als negativen Kompetenznormen und die Bestandsgarantie des Art. 79 III gegen sich hat, sondern auch die politische Realität: in welchem Bereich und in welchen Entscheidungen drückt sich denn die Herrschaft der (zahlenmäßigen) Mehrheit als empirisches Faktum und nicht nur als juristisch-politische Fiktion aus?

Der Rekurs auf das Prinzip demokratischer Selbstregierung könnte helfen, eine normative Leerstelle der verfassungs- und demokratietheoretischen Auseinandersetzung mit der Verfassungsgerichtsbarkeit zu schließen, die auch in den von Däubler/Küsel herausgegebenen Analysen noch offen bleibt. Zentraler Legitimationsgrund für die verfassungsgerichtliche Überprüfung von Gesetzen und der Maßstab für die Kritik an der Judikatur des BVerfG wird damit ein Grundsatz, den Rittstieg in seiner Untersuchung der ausländerrechtlichen Entscheidungen zu Recht in den Mittelpunkt rückt: der Minderbeitenschutz. Denn wenn es richtig ist, daß die Option des Grundgesetzes für eine parlamentarisch-repräsentative Demokratie nicht die Preisgabe des Grundsatzes demokratischer Selbstregierung des souverän gewollten Volkes bedeuten soll, dann läßt sich die Institutionalisierung von Mehrheitsentscheidungen verfassungssystematisch und demokratietheoretisch konsistent nur bei gleichzeitiger Verankerung des Minderheitenschutzes begründen. Volkssouveränität und Demokratie schließen nicht aus, daß politische Entscheidungen nicht einstimmig, sondern mehrheitlich gefällt werden können und von der Minderheit zu akzeptieren sind. Demokratisch legitimierbar und mit dem Prinzip der Volkssouveränität vereinbar sind solche Entscheidungsverfahren aber nur, wenn gesichert ist, daß Minderheiten die Chancen haben, Mehrheit zu werden, daß Minderheiten von der Mehrheit nicht tyrannisiert, politisch ausgebürgert oder sonstwie diskriminiert werden. Das zu verhindern ist vor- nehmster Zweck der verfassungsrechtlich verbürgten Freiheitsgarantien, deren Respektierung in letzter Instanz das Verfassungsgericht zu überprüfen hat. Dem BVerfG in Sachen Minderheitenschutz die Kompetenzen zu beschneiden oder ihm hier Selbstbeschränkung anzuempfehlen, würde dem Gericht also gerade die notwendige Legitimationsbasis entziehen.

\section{III.}

Schlothauers Untersuchung "Zur Krise der Verfassungsgerichtsbarkeit « versucht insofern, das Defizit kritischer empirischer Forschung abzuarbeiten, als sie einen systematischen Zusammenhang herstellen will zwischen Recht und Ökonomie im allgemeinen, der Entwicklung der staatsrechtlichen Methodenlehren und der gesellschaftlichen Entwicklung im besonderen sowie hauptsächlich zwischen den Legitimationsstrategien der neueren »sozialdemokratischen « Ansätze zur Methodik der Verfassungsinterpretation und der Legitimationskrise des Verfassungssystems im einzelnen. Man muß kein Prophet sein, um vorherzusagen, daß ein derart anspruchsvolles Unternehmen wie Schlothauers Studie fast zwangsläufig Kritik aus den unterschiedlichsten Richtungen provoziert.

Die Fragestellung und die in der Einleitung formulierten Prämissen ordnen Schlothauer in das Spektrum marxistischer Rechtstheorie ein. Allerdings läßt er frühzeitig erkennen, daß er den theoretisch gewundenen und empirisch steinigen Weg von der staatsrechtlichen Methode über das Recht zur Gesellschaft funktionalistisch abkürzen und durch die Konstruktion intentionaler Zusammenhänge überbrücken will. Zur Funktionalität des Rechts und der Methode erfahren wir: "Das moderne Recht ist nichts anderes als ein Aspekt, die Artikulation eines objektiven, wirklichen gesellschaftlichen Produktionsverhältnisses « (Cerroni). "Dies gilt auch für den Diskurs über das Staatsrecht und die staatsrechtliche Methodenlehre.« Aufgabe der Methode ist es, "die Rechtsnormen, die den Staat konstituieren ..., so mit der gesellschaftlichen Wirklichkeit zu vermitteln, daß diese gesellschaftliche Wirklichkeit, der die Methode ihre Entstehung verdankt, durch sie ihre Rechtfertigung findet. Dazu ist eine Methode aber immer nur so lange in der Lage, wie sie einer >wirklichen Wirklichkeit funktionelk (Cerroni) ist.« (II) Hinsichtlich 
des irritierend knappen staats- und rechtstheoretischen Rahmens, den der Autor dem Leser anbietet, wird er sich gegen den Einwand wappnen müssen, nicht zwanglos vereinbare Ansätze (J. Hirsch, S. v. Flatow/Huisken) verquickt zu haben, die im übrigen den von ihm postulierten Zusammenhang zwischen Reproduktionskrisen des Kapitals, Legitimationsproblemen des bürgerlichen Staates und endlich den Methodenkrisen (wer wollte einen solchen Zusammenhang bestreiten?) nicht so systematisch entfalten, daß ein kurzer Verweis auf diese Diskussion genügte.

Über die Intentionen der Methodentheoretiker behauptet Schlothauer: „Diese >Legitimationserschütterungen des Verfassungsstaates، (Kriele) sind es auch, die die Vertreter der neuen Ansätze ... auf den Plan riefen. « Und er verspricht "eine Analyse der Mittel derer (sie) sich . . bedienen, um (!) bestimmte verfassungsrechtliche Ergebnisse, die sich mit den herkömmlichen Methoden nicht begründen ließen, besser legitimieren zu können.» (13)

Im ersten Teil der Arbeit erläutert S. den Funktionszusammenhang von Gesellschaft und Methode exemplarisch an der Ablösung des staatsrechtlichen Positivismus Gerber-Labandscher Prägung durch den logischen Rechtspositivismus und Normativismus Kelsens und insbesondere durch die teleologische Methode von Thoma und Triepel. Seine Absicht ist, die These plausibel zu machen, daß sich dieser Zusammenhang »besonders deutlich in Zeiten großer sozialer und politischer Umbrüche und Krisen « zeige. Was sich ihm zeigt, ist der staatsrechtliche Positivismus als "Waffe des Bürgertums«: "zunächst zu seiner Machtausweitung gegenüber dem Adel « (33), dann zur Garantie des »ökonomischen Status quo..., in dem jede Gesetzgebung zugunsten der arbeitenden Klasse für unwirksam (erklärt werden) kann « (35). Man mag einwenden, diese ex-post-Einschätzung des staatsrechtlichen Positivismus sei doch ein wenig zu formelhaft geraten und nicht sonderlich originell.' Dem ließe sich entgegenhalten, daß hier keine historisch-systematische Analyse intendiert ist. Gewichtiger wären Einwände, die die Triftigkeit der Schlothauerschen Interpretation bestreiten,

\footnotetext{
5 Wenn man nicht ein anderes Interpretationsraster unterlegt, läuft man Gefahr, P. von Oertzens materialreiche Arbeit (Die soziale Funktion des staatsrechtlichen Positivismus, Frankfurt/M. 1974) nur zu verdoppeln.
}

diese zumindest stark modifizieren. ${ }^{6}$ Darauf ist weiter unten zurückzukommen.

Im Rahmen seines Ansatzes folgerichtig, geht Schlothauer im zweiten Abschnitt der "Methodenkrise « in der Rechtsprechung des BVerfG nach, ohne jedoch zunächst oder später die sozioökonomische Entwicklung der Bundesrepublik nach 1945 binreichend systematisch und deskriptiv darzustellen, obwohl er sie als Folie braucht, auf der die soziale Funktion der neueren staatlichen Methodik zu interpretieren wäre. ${ }^{7}$ Man wüßte gern genauer, welches die spezifischen gesellschaftlichen Bedingungen waren, die nach 1949 einer gesetzespositivistischen staatsrechtlichen Methodenlehre zu fröhlicher Urständ' verhalfen, und welche Veränderungen diesen Ansatz dann haben dysfunktional werden lassen. S. kontrastiert die Methoden»theorie « (?) des BVerfG mit dessen Methodenpraxis und findet heraus, daß das Gericht nicht tut, was es erklärt. Im einzelnen weist er dem BVerfG (a) Methodeninkonsistenz, (b) fragwürdige Methodeninnovation und (c) Methodenbruch, in totodaher "Rationalitätseinbußen " nach. Wer als Student der Judikatur des BVerfG je unternommen hat, sich auf den tieferen Sinn der Auslegung gemäß dem »objektivierten Willen des (Verfassungs-)Gesetzgebers « und auf ihr Verhältnis zur grammatischen oder systematischen oder teleologischen oder genetischen oder am Prinzip der Grundrechtseffektivität orientierten Interpretation einen logischen Reim zu machen, wird den Autor gewiß bestärken, den Vorwurf des »Methodenchaos« zu erheben. Auch die Folgerung, das Gericht habe »an den konkreten Ergebnissen seiner Entscheidungstätigkeit ein die Rechtsfindung leitendes Interesse", also nicht die Methode, sondern das Ergebnis regiere die Rechtsfindung ( 69 f.), erscheint weithin konsensfähig. ${ }^{8}$

6 Ladeur kritisiert die auch von Schlothauer vertretene Auffassung als "mechanistisches Verständnis des Kompromißcharakters des Verhältnisses von Adel und Bürgertum «, Verfassungsgerichtsbarkeit und politisches System, a. a. O., S.1 8 f., 23 f.; vgl. auch F. Hase, a. a. O., S. $103 \mathrm{ff}$.

7 Schlothauer sagt selbst, die gesellschaftliche Entwicklung Schlothauer sagt selbst, die gesellschaftliche Entwicklung
könne "nur unvollständig und schlagwortartig " (S. 96) dargelegt werden. Und in der Tat kann man keine sozioökonomische Realanalyse der letzten 100 Jahre erwarten, wohl aber - bei derart starken theoretischen Annahmen - eine solidere Basis für die Plausibilisierung des funktionalen Zusammenhangs.

$8 \mathrm{~K}$. Hesse, Grundzüge des Verfassungsrechts der Bundesrepublik Deutschland, 4. Aufl., Karlsruhe 1970, S. 22 ff. kommt zu einem ähnlichen Ergebnis, das er allerdings auf das Versagen der Interpretationsregeln zurückführt. 
Aber ist die Folgerung nicht fast trivial? Und wäre es nicht theoretisch riskanter (freilich auch zwingender) zu fragen: Erstens, ob das "Methodenchaos « Methode hat, ob das BVerfG bei seinen Entscheidungen im Spannungsfeld konfligierender und antagonistischer Interessen auf der Grundlage ihrer rechtlichen Übersetzung in eine Kompromißverfassung wie die des Grundgesetzes nicht gezwungen ist, ad hoc zu entscheiden, welcher der eingeklagten normativen Gesichtspunkte (»Rechtsgüter «) auf welchem Auslegungsweg als vorrangig abgesegnet werden soll? Zweitens, welche Prinzipien die Auslegung von Verfassungsnormen je nach Gegenstand und Kontext steuern? (Stattdessen festzustellen, das BVerfG habe den Boden "herkömmlicher Auslegungslehre " verlassen, wird auch durch die Berufung auf Forsthoff' nicht kritischer).) Wäre Schlothauer konsequent solchen Überlegungen nachgegangen, dann hätte er zwanglos die Funktionen des Verfassungsgerichts präziser fassen und sich fragen können, ob die Methodeninkrementalismus des BVerfG wirklich auf eine "Legitimationserschütterung" hindeutet oder ob das BVerfG, funktionsbewußt oder »legitimitätsschonend «, den gesellschaftlichen Status quo auf der dem nichtakademischen Publikum unzugänglicheren Ebene der juristischen Methode sanktioniert.

Für S. ist die "Legitimationserschütterung" des Verfassungsstaates notwendige Bedingung des Paradigmenwechsels in der staatsrechtlichen Methodenlehre, den er am Beispiel von Ehmke, Häberle, Kriele und $\mathrm{F}$. Müller diagnostiziert. Er untersucht die unterschiedlichen Legitimationsstrategien dieser Ansätze, deren gemeinsamer Nenner das Ziel sei, die Allgemeingültigkeit der im Wege der Verfassungsinterpretation gewonnenen Ergebnisse wiederherzustellen. In diesem gemeinsamen Ziel gehen für $S$. alle Differenzen auf: zwischen der topischen Methode (Ehmke) und der Rechtsgewinnung anhand vernunftrechtlicher Argumentation (Kriele); zwischen der Hermeneutik richterlicher Rechtserkenntnis (Kriele), dem Konsens aller Vernünftig-und-gerecht-Denkenden (Ehmke), einer materialen Wertordnung

9 E. Forsthoff, Die Umbildung des Verfassungsgesetzes, in: Festschrift für Carl Schmitt, Berlin 1969, S. 35 ff. stell zusammenfassend fest, »daß das BVerfG in den Vorstel lungen eines dem Verfassungsgesetz unterstellten und seine Innehaltung mit den Mitteln herkömmlicher Regelauslegungskunst sichernden Gerichts nicht mehr begriffen werden kann.« (S. 58 ). (so früher Häberle) oder einer Konsensprozedur (so Häberle heute) als Grundlage der jeweiligen Methode. Folgt man S., so ist es letztlich gleichgültig, ob die Methodik darin besteht, (a) durch genaue Beschreibung der juristischen Praxis, deren Gründe einsehbar zu machen (Kriele), oder (b) die Entscheidungsfindung in möglichst kleine Denkschritte aufzugliedern, das Vorverständnis offenzulegen, die Strukturen der Wirklichkeit empirisch zu ermitteln und hermeneutisch in den Rechtsfindungsprozeß einzuführen (F. Müller), oder (c) das Interpretationsverfahren zu demokratisieren (Häberle).

Diese Gleichgültigkeit resultiert aus deterministischen Festlegungen, die S. trifft: $\gg D i e$ -Interesselosigkeit der Juristen löst sich allemal kausal (!) in sozialen Determinanten auf « (Rottleutner). »Der >Common sense, an dem sich die Juristen zu orientieren vorgeben, ist also sozial determiniert. " ( 18 I f.) $)(D)$ ie sozialen Verhältnisse (lassen) keine Intersubjektivität in der Verwendungsweise (bestimmter Begriffe) zu «. Kurzum: Recht ist eben "Ausfluß bestimmter (!) gesellschaftlicher Interessen «, und Methodentheorien sind »Rechtfertigungslehren der Juristen für ihr eigenes Handeln «. ( $168 \mathrm{ff}$.) $\mathrm{Na}$ also.

Schlothauer ist zuzugeben, daß sich die Ablösung einer Methodenlehre nicht nur immanent aus deren Mängeln erschließen läßt. Unter dem Gesichtspunkt der sozialen Funktion solcher Lehren ist es daher notwendig, den »Methodenoptimismus « (I66) und jede Modernisierung der Verfassungsinterpretation auch ideologiekritisch zu durchleuchten. Wenn »Rechtsvernunft« (Kriele), »Sachgerechtigkeit« (Hesse), »Gemeinwohl« (Häberle), »herrschaftsfreie Diskussion « (F. Müller) abgelöst von ihrem gesellschaftlichen Konstitutions- und Verwendungszusammenhang thematisiert werden, sind die Einstiegsluken für die Vernünftigkeit der herrschenden Verhältnisse und für die Ideologie des Sachzwangs geöffnet. Und es ist zu fragen, ob die Rationalisierung des Vorverständnisses und des juristischen Entscheidens nicht am Ende auf der Ebene der Methode den gesellschaftlichen Status quo rationalisiert. Eine ganz andere Frage ist, ob man von den objektiven Funktionen einer Methodentheorie auf die subjektiven Intentionen des Methodentheoretikers schließen kann. Diese letzte Frage hat S. mit starken Vermutungen beantwortet. Er geht dabei nicht eben zimperlich mit den Vertretern der neueren staatsrechtlichen $\mathrm{Me}$ - 
thodik um: Er läßt durchblicken, daß sie »vielleicht nicht zufällig... politisch im Umkreis der Sozialdemokratie stehen " und meint daher, "die Ergebnisse der Verfassungsinterpretation, zu denen (sie) in konkreten Fällen kommen «, nicht wiedergeben müssen, "weil dies weitgehend einer Referierung herrschender sozialdemokratischer Politik gleichkäme«. (13) Außerdem behauptet er: "Den neuen Methodentheoretikern geht es also im Grunde um eine neue Legitimationsstrategie und nicht um eine stringente Methode«. (75) Spürt man nun arglos den Belegen nach, die Schlothauers Behauptung stützen sollen, dann erweisen sich einige als Belege dafür, daß es den Methodikern doch (und was in der Tat kritisch ist: nur) um eine stringente Methode geht. ${ }^{10} \mathrm{~S}$. darf sich auf den Einwand vorbereiten, er habe das hermeneutische Problem jeder Norminterpretation unterschätzt - und zwar wegen seiner Voreingenommenheit für die ausschließlich strategische Funktion jeder Methodik und wegen der den Methodikern unterstellten strategischen Intentionen.

Wenn man sich wie S. die anspruchsvolle Aufgabe stellt, den systemfunktionalen Stellenwert von Methodenlehren herauszuarbeiten, sollte man sich nicht noch zusätzliche und unnötige Beweislasten auferlegen. Wie will man schlüssig nachweisen, daß diese Ansätze "zu verhindern suchen, daß auch an ihnen das Verhältnis von Ökonomie und Recht untersucht wird «, daß "sie damit versuchen... zu verschleiern, welcher gesellschaftlichen Entwicklung sie ihre Entstehung verdanken und welche Funktion für welche gesellschaftlichen Verhältnisse sie zu erfüllen baben. « (170) Welchem Theoretiker wäre das hinreichend bewußt? Hat Schlothauer die herrschaftsstabilisierende Funktion theoretisch unzureichender und empirisch angreifbarer, vermeintlich marxistischer Kritik reflektiert? Hätte er das, dann wäre er etwa mit Häberle behutsamer umgegangen. So aber reduziert er dessen Legitimationsbegriff auf die Luhmannsche Verfahrenslegitimation und exemplifiziert seine Kritik zudem am untauglichen Objekt: Erstens, versteht Häberle die "Demokratisierung der Verfas-

\footnotetext{
- Hesse, a. a. O., geht es z. B. ausdrücklich um die »die Aufgabe richtiger Interpretation nach festen Grundsätzen« (S. 25). F. Miller fühtlion nach festen Grundsäschen Anspruch auf Methodenklarheit und Berechenbarkeit verpflichtet - vgl. dazu die Rezension von V. Neumann, KJ 1980, S. $337 \mathrm{ff}$
}

sungsinterpretation « im Sinne "qualitativer Einflußnahme der Beteiligten auf die Entscheidung « - und nicht nur als einen Mechanismus zur effektiven Verarbeitung von Enttäuschungen bei den Entscheidungsbetroffenen. Dazu hat Häberle in einem Aufsatz von 1975 ("Die offene Gesellschaft und ihre Interpreten) ${ }^{\mathrm{II}}$ Vorstellungen entwickelt, die S. nicht zur Kenntnis oder nicht ernst genommen hat. Zweitens, ist es schlichtweg falsch zu behaupten, Häberle setze in der Auseinandersetzung mit dem »Abhörurteil « seine neue Legitimationsstrategie ein, »um . . p praktisch zum gleichen Ergebnis zukommen wie das BVerfG« (196). Häberles Kritik liegt auf der Linie des Minderheitenvotums und geht stellenweise darüber hinaus. ${ }^{12}$ Ist das "praktisch das gleiche Ergebnis«?

Hätte Schlothauer sich seine deterministischen Vereinfachungen versagt, wäre er in der Lage gewesen, die Ambivalenz der neueren staatsrechtlichen Methodik zu analysieren und etwa den Ansatz von Häberle zu radikalisieren. Und die Herrschaftsvalenz dieser Methodenlehren hätte ihn nicht daran gehindert, ihre potentiell kritische Valenz freizulegen. Diese besteht darin, daß der objektivistische Schein richterlicher Rechtsfindung zwar nicht zerstört, aber (wenn auch personalistisch verkürzt) zum Problem wird, wenn von subjektiver Willkür, Vorverständnis etc. die Rede ist. Außerdem wird die Fiktion rechtlicher und richterlicher Neutralität angetastet, wenn die gesellschaftliche Wirklichkeit in die Interpretation einbezogen werden soll. Vor allem aber ist es Häberles Verdienst, der grassierenden Materialisierung der Verfassung (»Grundwerte«, »Wertordnung «) entschieden die Priorität von Verfahrensregeln und Entscheidungsprozeduren entgegengesetzt $\mathrm{zu}$ haben. Ist das nicht eine bedenkenswerte Position - wenn man davon ausgeht, daß gesellschaftliche Herrschaft sich über die Institutionen und auch über die Ideen und Handlungen der Juristen in rechtliche Entscheidungen vermittelt, daß also inhaltliche Festlegungen die Vermutung gegen sich haben, herrschaftsfunktional zu sein?

II JZ 75, $297 \mathrm{ff}$. Es ist schwer verständlich, warum SchlothZ 7s, anführt, dann aber nicht einarbeitet. So kritisierbar die anführt, dann aber nicht einarbeitet. So kritisierbar die
affirmative Bezugnahme auf die Poppersche Demokratiekonzeption auch ist, Luhmanns Legitimation durch Verfahren ist etwas anderes und anders zu kritisieren. I2 JZ 71,145 ff., bes. I 54 . 
IV.

F. Hase und K. H. Ladeur untersuchen in drei Studien charakteristische Merkmale, Brüche und Kontinuitäten in der deutschen Staats- und Verfassungsrechtslehre des 19. und 20. Jahrhunderts. Zum besseren Verständnis vor allem der ideologischen Entstehungs- und Funktionsbedingungen und der Funktionsweise der Verfassungsgerichtsbarkeit in der Bundesrepublik Deutschland analysieren die Autoren den Strukturwandel der Staatsrechtsideologie von der liberalen Verfassungslehre des Vormärz zum staatsrechtlichen Positivismus bis zu dessen Kritik durch die antiformalistische Staatslehre (Ladeur) und die Auseinandersetzungen über richterliches Prüfungsrecht und Staatsgerichtsbarkeit in Weimar (Hase); schließlich entwickelt Ladeur Vorüberlegungen zu einer Theorie des Verfassungsrechts, die er an einigen Urteilen des BVerfG illustriert. Der Leser wird im Vorwort von H. Ridder auf »schwere Kost « vorbereitet. Damit kann Hase nicht gemeint sein; seine Studie ist ein Beispiel dafür, daß auch eine lesbare wissenschaftliche Arbeit differenzierte Einsichten vermitteln kann. ${ }^{13}$ Im Hinblick auf Ladeurs Theoriesprache mutet das Prädikat "schwere Kost « bisweilen euphemistisch an. Da Hase und Ladeur trotz des gemeinsamen Erkenntnisinteresses sich unterschiedlichen theoretischen Traditionen verpflichtet fühlen und verschiedene Erklärungsstrategien verfolgen, erscheint es sinnvoll, sie getrennt zu rezensieren.

(I) Am Beispiel der Weimarer Republik will Hase die konkreten Bedingungen herausarbeiten, »unter denen justizielle Apparate zentrale politische Vorgänge und Kontroversen und vor allem die parlamentarische Gesetzgebung zu ihrem Gegenstand machen können " (104). Der Erklärung der kritischen Randbedingungen des justiziellen Diskurses in Weimar stützt Hase auf ein "Doppelstaats«theorem: »Der Begriff >Doppelstaatlichkeit ... . bezeichnet einen krisenhaften Gegensatz der staatlichen Apparate", "eine Phase, in der zwei Instanzen - die Legislative und die zentrale Exekutive - um die Dominanz im Staat rivalisieren « $(\mathrm{I} 0 \mathrm{~S}){ }^{14}$ In einer solchen

I3 Das gilt im übrigen auch für Ladeurs Beitrag in Däubler/Küsel: Die Meinungsfreiheit in der Rechtsprechung des BVerfG) S. IO2 ff., wo er anschaulich und schlüssig nachweist, daß in den Entscheidungen des BVerfG ein nachweist, daßs in den Entscheidungen des BVerfG ein
substanzhaftes, nicht prozedurales Öffentlichkeitskonzept dominiert.

14 Hase verwendet die Kategorie anders als E. Fraenkel
Krise, so Hase, können sich die staatsrechtlichen Ideologen nicht mehr problemlos auf die Praxis des Staates beziehen, weil eine einheitliche Praxis als Bezugspunkt fehle. Hase demonstriert dies an der Kontroverse über die Frage, ob die Justiz ein allgemeines Recht haben sollte, Gesetze auf ihre Verfassungsmäßigkeit zu überprüfen, d. h. ob jedes Gericht in jedem Streitfall befugt sein sollte, diese Prüfung vorzunehmen und ggf. Gesetze außer Anwendung zu lassen; oder ob die Prüfungskompetenz bei einem zentralen Verfassungsgericht konzentriert sein sollte. Das allgemeine Prüfungsrecht weist der Justiz einen »distinkt doppelstaatlichen "Status zu: sie ist aus ihrer strikten Rechtsanwendungsfunktion herausgelöst und der Legislative als eigenständige politische Instanz gegenübergestellt. Diese »doppelstaatliche « Justizpraxis ist durch den Widerspruch charakterisiert, sich als Rechtsanwendung darstellen zu müssen. Demgegenüber ist nach Hase das konzentrierte Prüfungsrecht bei einem Verfassungsgericht als "pluralistischer "Instanz institutionalisierbar. Deren Funktionsvoraussetzungen wären (a) die Vorstellung, daß (parlamentarische) Minderheiten eigene Rechte an der Gesetzgebung geltend machen können, (b) ein Konsens der politischen Parteien, gesellschaftlichen Gruppen und staatlichen Organe, verfassungsgerichtliche Entscheidungen als bindend $\mathrm{zu}$ akzeptieren, und (c) eine konsistente und einheitliche Verfassungsideologie als Bezugsgröße für die Entscheidungsregeln des Verfassungsgerichts. Insbesondere die letzte Voraussetzung sieht Hase in Weimar als nicht erfüllt an. Die »Doppelstaatlichkeit« der Praxis äußert sich darin, daß Begriffsbildung und Theorie der konservativen und der parlamentarisch-pluralistisch orientierten Staatsrechtslehre antithetisch aufeinander fixiert sind und an rivalisierenden Integrationskonzepten festhalten. Weder für den repressiven Etatismus der Konservativen doch für den integrativen Etatismus der Liberalen ist die positive Verfassung Grundlage verbindender Ordnungsvorstellungen. Erstere rekurrieren auf das substanzhaft-hermetische an der Praxis der Exekutive orientierte Integrationsideal; aber auch die parlamentarisch-liberale Lehre

(Der Doppelstaat, dt. Rückübersetzung, Frankfurt/M.-Köln 1974), dessen Gegenüberstellung von Normenstaat und Maßnahmestaat nach Hases Auffassung auf einer unkritischen Vorstellung des überkommenen Rechtsstaates beruht.
447 
448 vermag die Legitimität nicht selbst zu besetzen und verweist sie pauschal aus dem positiven Recht.

Hases sorgfältige Rekonstruktion der konkurrierenden Ordnungsideologien und deren wohltuend differenzierte funktionsanalytische Einschätzung sind plausibel auf die Struktur staatlicher und insbesondere justizieller Praxis bezogen. Diese Struktur bleibt jedoch theoretisch unterbestimmt. Das Konzept der »Doppelstaatlichkeit« überzeugt als heuristisches Modell. Es bleibt jedoch unvollständig, solange über die vorausgesetzte (und voraussetzungsvolle) einheitliche Staatspraxis nicht mehr ausgesagt wird, als daß sie notwendige Bedingung einer einheitlichen Verfassungsideologie ist. Wenn nicht auch - etwa im Rahmen eines Hegemoniekonzepts außerstaatliche und nicht-ideologische Faktoren eingeführt werden, die eine einheitliche Staatspraxis erzwingen oder vereiteln, bleibt die Analyse zu sehr auf die Mechanismen innerstaatlicher Arbeitsteilung und Ideologieproduktion fixiert, also etatozentrisch und vernachlässigt die gesellschaftlichen Entstehungs- und Funktionsbedingungen von Staats- und Verfassungsideologien.

(2) Verfassungsgeschichte und die Entwicklung von Verfassungsideologien können nach Ladeurs Auffassung zureichend weder immanent kritisiert noch durch die funktionsanalytische »Enthüllung « der hinter den juristischen Konstruktionen stehenden Klasseninteressen noch durch eine Theorie der sozialen Evolution erklärt werden. Für zureichend hält Ladeur eine materialistisch fundierte strukturalistische Theorie der Ideologiebzw. Diskursformation, die insbesondere die verfassungstheoretischen Diskurspraktiken innerhalb einer Gesellschaftsformation als Forschungsgegenstand thematisiert und ihren Gegenstand als "regionale Wissenschaft « konstruiert; d. h. vier "Regionen« wissenschaftlicher Erkenntnis in einen einheitlichen theoretischen Rahmen einbaut: »Erstens eine materialistische Theorie der Gesellschaftsformationen und ihrer Transformationen einschließlich ihrer Ideologien, zweitens sprachwissenschaftliche Konzeptionen einer begrifflichen Rekonstruktion von Aussagevorgängen ..., drittens eine Theorie der historischen Bestimmung semantischer Prozesse und schließlich viertens eine psychoanalytisch informierte ,Querschnitttheorie der Subjektivität«. (2I f.) »Eine Theorie der Diskursformationen, die determinieren, was, wie, von wem innerhalb einer bestimmten historischen Konjunktur gesagt werden kann, sowie die Analyse ihrer sei es antagonistischen sei es Allianz- und Dominanzverhältnisse hätten eine Verfassungsgeschichte zu ermöglichen, die das Subjekt eines historischen Aussageprozesses nicht mehr in der rreferentiellen Illusion ‘ der für sich selbst sprechenden Sprache zum Verschwinden brächte, sondern es vielmehr innerhalb seines Textes selbst auftreten ließe und so die Materialität der Sprache als eine in gesellschaftliche Widersprüche involvierte und intervenierende Praxis begrifflich zu rekonstruieren hätte« (22), fordert Ladeur.

Wer sich von diesem gigantischen Anspruch nicht abschrecken und sich von so viel spätbildungsbürgerlicher Gelehrsamkeit nicht entmutigen läßt, erkennt hinter dem Begriffszauber das Vorhaben, die losen Stränge unterschiedlicher Theorietraditionen miteinander zu verknüpfen. Man fragt sich allerdings, ob Ladeur hoffen kann, die Einbruchstellen der jeweiligen Theorien durch ein richtiges Verständnis und die richtige Kombination zu überqueren. Das prätentiöse Theorieprogramm scheint den Theoretiker selbst zu erschrecken: Im Hinblick auf die Leistungsfähigkeit äußert er sich vorsichtig; sodann erkennend, daß es sich gleichzeitig auf mehreren theoretischen Hochzeiten empirisch nicht gut tanzen läßt (allein die Pflichttänze reichen für eine Serie von Habilitationen), entledigt sich der Autor einiger der Verpflichtungen, die er sich zuvor aufgeladen hat: "Dies kann hier nicht weiter vertieft werden «, heißt es an mehreren kritischen Punkten der Arbeit. ${ }^{\text {Is }}$

Man würde allerdings dem Wert der Ladeurschen Studie nicht gerecht, wenn man ihn an der Zahl der salvatorischen Klauseln abläse. Denn sein komplexer, wenngleich noch sehr eklektizistisch wirkender Ansatz gestattet ihm, die gleichen Phänomene sehr viel differenzierter $\mathrm{zu}$ interpretieren als (womöglich noch deterministisch verengte) Funktionsanalysen. So hebt er etwa in bezug auf den staatsrechtlichen Positivismus, die Staatsideologie der "passiven Revolution « des Bürgertums, zu Recht nicht den Bruch in der wissenschaftlichen Kontinuität« (von Oertzen), sondern die in der Absage an die

is Vgl. S. 23, 24, 25, 26, 38, 67, 219, 234, 257, 260, 279, 304. 
Volkssouveränität gewahrte Kontinuität zur älteren deutschen Staatslehre hervor. Indem Ladeur sich nicht auf die "Enthüllung « der hinter solchen Ideologien stehenden Interessen verlegt, den staatsrechtlichen Diskurs nicht nur als Reflex, sondern auch als Gegenstand gesellschaftlicher Machtkämpfe begreift, bilden die liberale Verfassungslehre des Vormärz, der staatsrechtliche Positivismus Gerber-Labandscher Provenienz und dessen antiformalistische Kritik eben nicht nur passiv jeweils neue Legitimationsideologien $a b$; vielmehr werden in den Verschiebungen zwischen Rechtssubstantialismus und Rechtsformalismus gesellschaftliche Veränderungen aktiv ausgetragen.

Seine Vorüberlegungen zu einer Theorie des Verfassungsrechts ( 189 ff.) entwickelt Ladeur an dem Paradigma der "grundlegenden Konvention « (Negri) der bürgerlichen Gesellschaft als Modell gesellschaftlicher "Normalität«. Diese Konvention bindet in der bürgerlich-parlamentarischen Demokratie die Legitimation eines jeden Gesetzes an die Zustimmung des Souveräns im Wege eines demokratischen Entscheidungsverfahrens. Nachdem Ladeur eben noch und zu Recht immanenten Kritikern des BVerfG, die von "Grenzüberschreitungen " reden, vorgeworfen hat, ein normativ überhöhtes Modell der Normalität des parlamentarischen Systems der Bundesrepublik vorauszusetzen, behauptet er nun seinerseits: Die Funktionsweisen von Parlament (Zustimmung) und Exekutive (Unterwerfung unter Ordnungsideologien) seien historisch durch eine "grundlegende Konvention « koordiniert gewesen, welche die gesellschaftliche Kontraktverfassung als "relativ konsistentes Funktionsmodell bürgerlicher >Normalität< insgesamt voraussetzen konnte«, (202 f.) Seine These ist nun, die Voraussetzungen dieses Funktionsmodells seien im Übergang zum staatsinterventionistischen Kapitalismus entfallen: Das Rechtssubjekt als Bezugsgröße staatlichen Handelns habe sich verflüssigt, seine Verhaltens- und Bedürfnisstruktur sei keine kalkulierbare Größe mehr, sondern bedürfe der Planung; der Funktionsmodus der Zustimmung werde vom Verfahren der Gesetzgebung abgelöst und auf das politische und gesellschaftliche System insgesamt bezogen. Im Formwandel des Rechts - Verflüssigung des Rechtssubjekts, Auflösung des Vertrages, Transformation der allgemeinen Gesetze in konkrete Regelungen, "die die Rekonstruktion der tatsächlichen politischen und ökonomischen Voraussetzungen der Ordnung durch eine Tieferlegung der juristischen Abstraktion zu leisten versuchen « $(224 \mathrm{f}$.) - erkennt Ladeur den Identitätsverlust des Staates als des universellen Subjekts und den Zerfall der bürgerlichen Ordnung.

Es sei dahingestellt, ob damit die frühbürgerliche Ordnung auf ihren empirisch ausweisbaren Rechtsbegriff gebracht ist. Allerdings leuchtet ein, daß die Entwicklung zum entformalisierten strategischen Recht einerseits und die Verstärkung der symbolischen Funktion von Recht sich heute nicht mehr angemessen als Verschiebung vom Konditionalzum Zweckprogramm kennzeichnen läßt. Aus der Veränderung der Gesetzesform ergeben sich veränderte Bedingungen und Funktionen verfassungsgerichtlicher Kontrolle. An die Stelle der Selbstkontrolle des Staates durch seine eigenen Rechts-Gesetze tritt nun die im BVerfG institutionalisierte »Kontrolle des Staates durch den Staat«. Nach dem Zerfall eines im Parlament gestifteten Konzepts von Allgemeinheit wird das Gesetz kontingent gesetzt und durch ein abstraktes Symbol, die Rechtsverfassung, über die das BVerfG wacht, überhöht. Bürgerliche Freiheit und bürgerliche Ordnung werden in ein Rechtsverhältnis überführt, das in der gerichtsförmigen "Abwägung « inszeniert werden muß, weil die ideologische Reproduktion des Verhältnisses von Verfahren der Zustimmung und Unterwerfung in der Praxis der Staatsapparate nicht mehr funktioniert. Die Hauptfunktionen des BVerfG bestehen daher in der Kontrolle und Vereinheitlichung der herrschenden Ideologieformation und in der Herstellung der ideologischen Kohärenz der Strategien der Staatsapparate.

Die Funktionsweise und die institutionellen Darstellungsbedingungen der Rechtsprechung des BVerfG in seiner »organisationalen Umwelt« untersucht Ladeur abschließend exemplarisch in konkreten Konfliktfeldern. Die "provisorischen " (Ladeur), sehr verkürzten Urteilsanalysen sind in mehrfacher Hinsicht unbefriedigend. Zum einen sind sie so kurz und so wenig konkret, daß von Analyse im strengen Sinne nicht die Rede sein kann, wenn etwa die Entscheidung zum $\ 2$ I 8 auf neun und der "Radikalen «beschluß auf vierzehn Zeilen rhapsodisch abgehandelt werden. Zum anderen ist die Auswahl der Urteile nicht repräsentativ genug, um der interessanten These Ladeurs, das BVerfG erfülle seine 
Hauptfunktion in der constituentpolicy-Arena, d.h. positiv durch Symbolpflege und Konsensstiftung, negativ durch die Nichtthematisierung vor allem von grundsätzlichen Problem der Redistribution und durch die Stigmatisierung von "Systemfeinden « zur Sicherung der Bestandserhaltung des politischen und ökonomischen Systems, mehr als eine oberflächliche Plausibilität zu verschaffen.

Die Defizite der Urteilsanalyse werden besondes deutlich, wenn man anhand des Mitbestimmungsurteils Ladeurs Kurzkritik (312f.) mit einer ebenso detaillierten wie einleuchtenden Analyse vergleicht. ${ }^{16}$ Ladeur sieht das BVerfG in der Zwangslage entscheiden zu müssen (also nicht einfach den Streit an den Gesetzgeber zurückweisen zu können), aber gleichzeitig ein negatives Urteil gegen die beteiligten organisierten Interessen kaum durchsetzen zu können: »In dieser Zwangslage hat das Gericht versucht, sozusagen ein Rechts-»chip « mit unbestimmtem Wert, aber mit bestimmter Schwankungsbreite auszuspielen: das Gericht macht die Zulässigkeit der bestehenden Variante unter-paritätischer Mitbestimmung vom loyalen Gebrauch dieser Rechte abhängig." (313) Mückenberger stellt demgegenüber zunächst fest: "(Das BVerfG) hat sich mit einer argumentativen Eleganz - die mir Staunen und Respekt abverlangt - dem ihm von beiden konfligierenden Parteien angemuteten $\mathrm{Er}$ wartungsdruck entzogen, auch nur irgendeine Andeutung über die $>$ Reformfähigkeit dieser parlamentarischen Demokratie zu machen. (49 f.) Und weiter: "Gewiß enthält das Urteil die Anregung einer Korrektur durch den Gesetzgeber, falls sich die der Mitbestimmung gestellte Prognose als falsch erweisen ... und zukünftig die Funktionsfähigkeit der Unternehmen ... durch die Mitbestimmung beeinträchtigt werden sollte. Aber diese Anregung ist einerseits trivial, weil Selbstverständliches aussprechend, zum anderen mangels objektivierbarer Voraussetzungstatbestände nicht justiziabel.« (52) Der eigentliche Kern der Mitbestimmungsentscheidung wird bei Ladeur nicht, bei Mükkenberger schlüssig herausgearbeitet: die Verknüpfung eines extrem individualisti-

I6 U. Mückenberger, Mitbestimmung und „Funktionsfähigkeit« der Unternehmen. Zum Mitbestimmungsurtei des Bundesverfassungsgerichts vom I. März 1979, in: Däubler/Küsel, a. a. O., S. $49 \mathrm{ff}$. schen Grundrechtsverständnisses über das „Verhältnismäßigkeitsprinzip« mit der den gesellschaftlichen Zusammenhalt garantierenden Grundnorm der "Funktionsfähigkeit« ( $s 9 \mathrm{ff}$.).

Aus diesem Vergleich folgt keineswegs, daß Ladeurs komplexe theoretische Konzeption nicht durchzuführen oder unergiebig ist; gleichwohl muß sie noch unter Beweis stellen, daß die theoretische Komplexität nicht um den Preis einer unangemessenen Stilisierung der Empirie erkauft wird, und daß ein solcher Ansatz mehr Erkenntnisgewinn einbringt als weniger aufwendige, aber dennoch aufgeklärte Varianten marxistischer Rechtskritik.

Günter Frankenberg

Joachim Perels (Hrsg.), Grundrechte als Fundament der Demokratie, Frankfurt am Main 1979, 263 Seiten, DM II,-(edition subrkamp 95I)

$\mathrm{Zu}$ den Verfassern der Einzelbeiträge des Sammelbandes zählen vier Öffentlich- und ein Arbeitsrechtler, drei Politikwissenschaftler und als einziger Vertreter forensischer Praxis der Münchner Rechtsanwalt Ott. Die Fachbereiche Rechts- und Politikwissenschaft der Universität Hannover stellen allein fünf Autoren. Die knappe Mehrheit der Verfasser gehört dem Mitarbeiterkreis bzw. dem Redaktionskollektiv der »KJ « an. Schon die Namen der Autoren geben Anlaß, zwei allgemeine Bemerkungen vorauszuschicken, die die Einordnung der Beiträge in einer sich als kritisch verstehenden Rechtswissenschaft betreffen.

Wie jede wissenschaftliche Praxis bestimmt sich auch die Rechtswissenschaft im Spannungsfeld der Pole Konstruktion und Kritik. Die Arbeiten linker Juristen der 7oer Jahre zeichneten sich durch eine deutliche Schwerpunktsetzung zugunsten der Rechtskritik aus. Nun ist Arbeitsteilung in einer Wissenschaft nicht von vornherein schädlich, vor allem dann nicht, wenn eine etablierte Wissenschaft dazu neigt, Kritik auf die der h. M. beigesellte a. A. zu reduzieren. Fatal wird die Arbeitsteilung zwischen Konstruktion und Kritik, wenn die Kritik sich bewußt vom fachwissenschaftlichen Diskurs abkoppelt, indem sie die konstruktive Arbeit, und für die 
Juristen heißt dies immer auch: die rechtsdogmatische Arbeit, den jeweils anderen überläßt und sich selbst damit begnügt, die Ergebnisse dieser Arbeit von einem fachtranszendenten Standpunkt aus zu zerpflükken. Es verwundert nicht, daß die Fachwissenschaft auf eine solche Kritik gereizt reagiert bzw. sie überhaupt nicht mehr zur Kenntnis nimmt. Umgekehrt kann die Abkoppelung von der konstruktiven Arbeit der Fachwissenschaft zu einer gefährlichen Verengung der Methoden und Erkenntnisinteressen der Rechtskritik führen. Kritik wird fundamentalistisch, wenn sie nach einem archimedischen Punkt sucht, von dem aus die Welt der etablierten Wissenschaft aus den Angeln gehoben werden soll. Perels hat in seinem Vorwort auf knappem Raum letztere Tendenz vorsichtig angesprochen. Der eindeutige Verdienst des Sammelbandes besteht nun darin, daß er eine sichtbare Zäsur gegenüber solchen Tendenzen markiert. Die Beiträge lassen sich lesen als Versuch, die leidige Arbeitsteilung zwischen konstruktiver und kritischer Rechtsarbeit aufzuheben.

$\mathrm{Da} ß$ das Interesse an konstruktiver Arbeit auch auf die Schärfe und Treffsicherheit der Rechtskritik positive Auswirkungen haben kann, beweist der Aufsatz v. Brünnecks zu den Enteignungs- und Entschädigungstheorien des Art. 14 GG. Zunächst läßt der dem Aufsatz vorangestellte Abschnitt Schlimmes befürchten. Die Rechtsprechung zur Eigentumsgarantie solle nach den "politischen und gesellschaftlichen Interessen «, die "hinter" den Interpretationen stehen, befragt werden. Die "rechtspositivistisch verengte Normlogik « sei zu sprengen, ". . . auch wenn dieser Anspruch angesichts des gegenwärtigen Diskussionsstandes nur begrenzt einzulösen ist «. (S. 215). Der solchermaßen strapazierte Rezensent neigte dazu, seiner zwischenzeitlich insoweit gefestigten Vorurteilsstruktur nachzugeben und die folgenden Seiten zu überschlagen. Dies wäre bedauerlich gewesen, denn dem unglücklichen Vorspann folgt gerade nicht der ideologiekritisch erhobene Zeigefinger, auch nicht die Versicherung einer brüchigen Objektivität vermittels politökonomischer Ableitungsakrobatik, sondern eine feinziselierte Analyse der Dogmatik zu Art. I4 GG. Die rechtspolitischen Folgerungen gehören nicht $\mathrm{zu}$ jenen politischen Setzungen, die schon vor jeder Analyse irgendwie gewußt werden, sondern sind analytisch belegte und sorgfältig geprüfte Aussa-

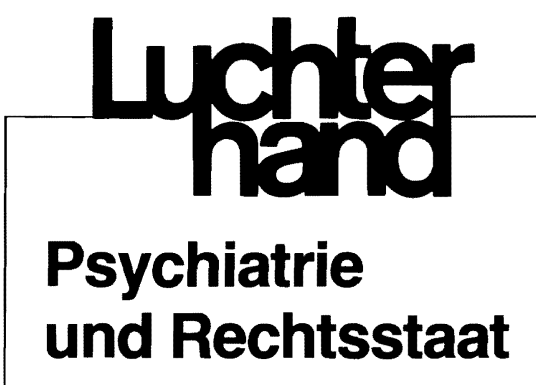

Herausgegeben von Manfred Bergener mit Beiträgen von

Franz Andritsch, Manfred Bergener, Hans J. Bochnik, Helmut J. Gärtner, Hanfried Helmchen, Gerhard Hopf, Dirk Lorenzen, Gerhard Möllhoff, Carlo Nässig, Wolfgang Pittrich, Fritz Reimer, Werner Richtberg, Hans-Ludwig Schreiber, Ulrich Venzlaff, Arnold Wiebe Reihe: Demokratie und Rechtsstaat, Band 54

251 Seiten, kartoniert, DM 34,ISBN 3-472-08027-2

Psychische Krankheit ist nicht das Problem einer verschwindend geringen Minderheit: jährlich bedürfen mehrere Millionen Menschen in der Bundesrepublik einer psychiatrischen Behandlung. Während psychische Krankheiten weit mehr als körperliche Erkrankungen durch soziale Umstände hervorgerufen, gefördert und geprägt werden, vollzieht sich die ärztliche Behandlung vielfach in der Isolation langdauernder anstaltlicher Unterbringung. Schutz der Gesellschaft vor dem Kranken, Schutz des Kranken vor sich selbst standen und stehen oft noch im Vordergrund der rechtlichen Bewältigung psychischer Krankheit. Der Kranke selbst gerät zumeist in eine Situation, in der seine Außerungen und Forderungen nicht mehr ernstgenommen werden, in der er weitgehend wehrloses Objekt rechtlicher Entscheidungen wird. Die Reformbestrebungen der letzten Jahre verlangen auch eine kritische Überprüfung der rechtlichen Aspekte. 
gen. Geronnenes wird verflüssigt, die Theorienbündel zu Art. I4 GG öffnen sich dem Verstehen und der reflexiven Aneignung. Jedem Studenten, der an den trockenen Lehrbuch- und Repetitoriendarstellungen der Enteignungsdogmatik zu verzweifeln droht, sei die Lektüre dieses Aufsatzes ans Herz gelegt. Die zitierten Sentenzen aus dem Einleitungsabschnitt sind also sachlich irreführend, suggerieren sie doch, daß die vorgelegte Rechtskritik nur von einem "Standpunkt außerhalb der Rechtswissenschaft geleistet werden konnte. Brünnecks Analyse erstellt selbst den Gegenbeweis, indem sie das fachwissenschaftliche Kritikpotential gewissermaßen ausreizt.

Die Überlegungen zum Verhältnis von Fachund Nachbarwissenschaft leiten über zu einer zweiten Anmerkung, die sich dem Rezensenten aufdrängte. Die Autoren scheinen insgesamt um eine kritische Modifizierung des Verhältnisses von Rechts- und Sozialwissenschaften bemüht zu sein. Interdisziplinarität kann und sollte m. E. heißen, fachwissenschaftliche Reflexion bis an jenen kritischen Punkt zu treiben, von dem aus wissenschaftliche Erkenntnis nur noch im Rekurs auf Nachbarwissenschaften möglich wird. Betrachtet man die im letzten Jahrzehnt vorgelegten Arbeiten überwiegend sozialwissenschaftlich orientierter Juristen, kann man sich des Eindrucks nicht ganz erwehren, daß Interdisziplinarität materialisiert wurde. Eine neue Wissenschaft, irgendwo und irgendwie zwischen Jurisprudenz und Sozialwissenschaften angesiedelt, schien erstanden zu sein, eine Wissenschaft, die eigene Themen und Methoden entwickelte. Fragwürdig an diesem Begriff von Interdisziplinarität ist seine nahezu perfekte Immunisierung. Gegenüber der Soziologie und Politologie ist die »interdisziplinäre Wissenschaft « Jurisprudenz, vor der juristischen Kritik zieht sie sich in das sicher geglaubte Terrain der Sozialwissenschaften zurück. Es ist ähnlich wie beim Wettlauf des Hasen mit Swinegel. Gelangt der sozialwissenschaftliche Hase an das Ziel seiner Bemühungen, springt $S$ winegel aus der juristischen Furche: "Ich bin allweil hier.» Dem juristischen Hasen blüht das gleiche Schicksal, Swinegel ist »immer schon « da. Falsch an dem Bild ist die Unterstellung, die Fachwissenschaften würden zu dem Wettlauf antreten, richtig daran könnte sein, daß Swinegel nicht vorankommt.

Von dieser - zugegeben verkürzt und nicht ohne polemische Absicht dargestellten Interdisziplinarität unterscheidet sich Perels Buch wohltuend. Befreiend ist etwa Keßlers nüchternes und methodenbewußtes Statement, die sozialwissenschaftliche Widerlegung der gesellschaftlichen Prämissen einer konservativen Verfassungsinterpretation beinhalte noch keine Aussage über den streitigen Inhalt der konkreten Verfassungsnorm. Alle Autoren setzen sich der - wie Perels im Vorwort schreibt - »konkreten Auseinandersetzung mit Verfassungsinterpretationen « aus und knüpfen dabei an den fortgeschrittensten Stand juristischer Theoriebildung an.

$\mathrm{Daß}$ der Titel des Buches programmatische Zielsetzung ist, zeigt das Vorwort des Herausgebers in dem an die Einzelbeiträge herangetragenen Anspruch, »exemplarisch Elemente einer demokratischen Grundrechtsinterpretation zu entwickeln «. (S. 8). Nun werden Grundrechtsinterpretationen auch von einem grundrechtstheoretischen Vorverständnis geleitet, über dessen verfassungsrechtliche Legitimität seine verfassungstheoretische Begründbarkeit mitentscheidet. $\mathrm{Pe}$ rels implizite Bezugnahme auf eine demokratische Grundrechtstheorie macht stutzig, nachdem spätestens seit 1973 in der verfassungstheoretischen Diskussion jener Richtung im deutschen Staatsrecht, der die Autoren sich überwiegend zugehörig fühlen dürften, die freiheitssichernden Formalgarantien des Rechtsstaates besonders betont werden. Die Stichworte "zweistufige Legalität" und "Reformalisierung des Rechtsstaates « mögen als Hinweise genügen. Eine rechtsstaatlich orientierte Verfassungstheorie wird die Grundrechte zuvörderst als Abwehrrechte begreifen und eher dem klassischen Eingriffsund Schrankendenken verpflichtet sein.

Einem Mißverständnis sei vorab begegnet. Gewiß ist es ein ideologieträchtiges Geschäft, auf der Ebene der Verfassungstheorie rechtsstaatliche und demokratische Strukturelemente gegeneinander auszuspielen. Kaum weniger inkonsistent aber ist die Behauptung einer harmonischen Einheit von Rechtsstaats- und Demokratieprinzip. Aufgabe einer jeden Verfassungstheorie ist es, beide Strukturprinzipien zu verbinden. Während auf der Ebene der Verfassungstheorie die Vermittlung ein Problem der vorsichtigen Akzentsetzung bleibt, wird im Vorgang der Grundrechtsinterpretation die Frage nach der Gewichtung der Prinzipien vor die Entscheidung gestellt. Denn die Ergebnisse der Kon- 
kretisierung von Grundrechten werden unterschiedlich ausfallen, je nachdem ob der Interpret die rechtsstaatlichen oder die demokratischen Akzente der Verfassungstheorie in die Grundrechtstheorie überführt.

Schneiders Beitrag, der den verfassungsgeschichtlichen sowie den verfassungs- und grundrechtstheoretischen Rahmen für die Einzelstudien entwirft, und der auch grundrechtspolitisch argumentierende Aufsatz Abendroths sind spannend zu lesen aufgrund der augenfälligen Divergenz in der Behandlung der grundrechtstheoretischen Problematik. Abendroth läßt der scharfen Kritik an Naturrechtsideologien und Wertphilosophien in der Rechtswissenschaft und -praxis ein Plädoyer zugunsten der Grundrechte als "Verteidigungschancen des freiheitlich demokratischen Rechtsstaates für die Rechte des einzelnen Bürgers" (S. 258 ) folgen. Die in der Aufforderung, "Grundrechte im Zweifek stets zugunsten des möglichst breiten Gestaltungsspielraums des individualisierbaren Rechtssubjekts auszulegen «, angezeigte klare Entscheidung für die rechtsstaatliche Formseite der Grundrechte erstellt das Fundament, auf dem eine verfassungstheoretisch stimmige Vermittlung von Grundrechtssystem und Demokratie möglich wird. Um der Erhaltung und des Ausbaus der Demokratie willen ist die Freiheitssphäre des einzelnen Bürgers mittels jener zwecklos-formalen rechtsstaatlichen Formtypik zu schützen, an der im Konfliktfall auch Eingriffe des demokratischen Gesetzgebers scheitern.

Schneider hingegen scheint die Problematik mit dem Satz »Wir wollen alles« lösen zu wollen. Für ihn haben Grundrechte eine demokratische, rechtsstaatliche und sozialstaatliche Funktion. Die Grundrechte gewährleisten nicht nur Freiheitsräume und normieren Eingriffsverbote, sie sind auch nicht nur sozialstaatliche Leistungsrechte und demokratische Teilhaberechte, sondern sind zudem "Elemente objektiver Ordnung" und normieren - über den sozialen Leistungsaspekt hinaus - »Pflichten des Staates «. Die Summierung der grundrechtstheoretischen Triade mit dem materialen Rechtsstaatsbegriff soll sodann für die Grundrechtsinterpretation mittels der Einführung des Prinzips der »funktionalen Mehrdimensionalität der Grundrechte « fruchtbar gemacht werden. Daß die Überführung dieser Grundrechtstheorie in Grundrechtsdogmatik nur zum Preis der Abdankung rationaler Metho-

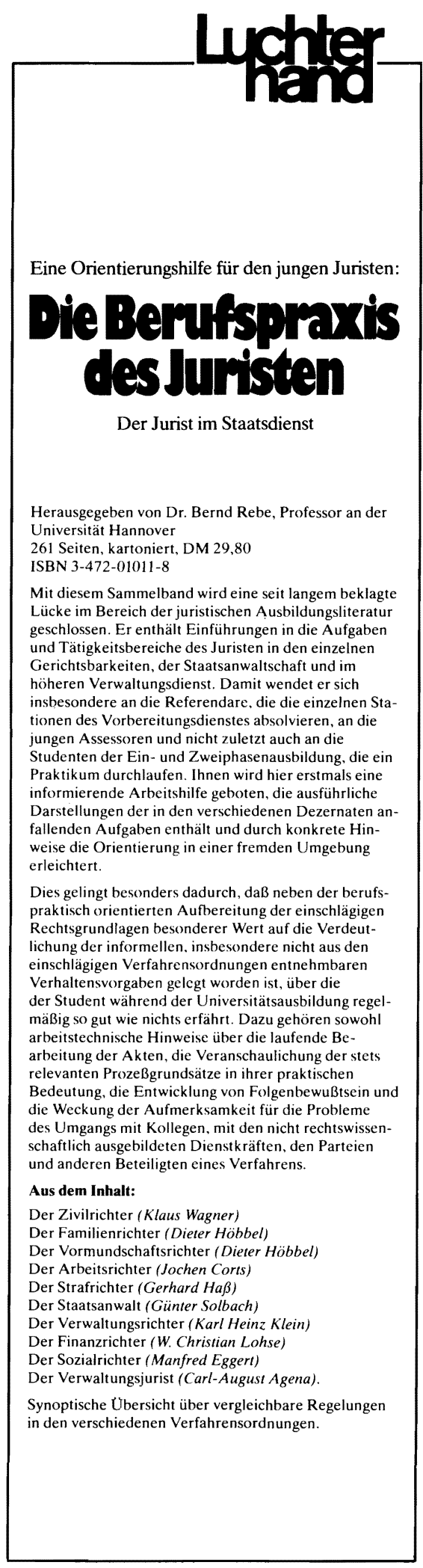


dik zu haben ist, belegt der Verfasser am Beispiel der Pressefreiheit (S. 35 f.). Gleichberechtigt und unverbunden steht ein subjektives Abwehrrecht der Presseangehörigen gegen staatliche Eingriffe neben einem Anspruch an den Gesetzgeber auf Gewährleistung der inneren Pressefreiheit. Wie das Eingriffsverbot mit der Eingriffsermächtigung im Konfliktfall methodisch vermittelt werden kann, bleibt ungeklärt. Der schillernde Begriff der "Funktion" verdunkelt das Problem mehr, als daß er eine Lösungsstrategie aufzeigte: in welchem Sinne und zu welchem Zwecke soll die apostrophierte Mehrdimensionalität »funktionieren«? Soll die Antwort nicht dezisionistisch ausfallen, werden weitere Kriterien bemüht werden müssen. Verfassungsrechtlich legitim aber können diese Kriterien nur dann sein, wenn sie auch in einer Verfassungstheorie begründet sind. Die Prioritätensetzung, der Schneider im Bereich der Grundrechtstheorie ausweicht, holt ihn auf der Ebene der Verfassungstheorie wieder ein. Man kann grundrechtstheoretisch zwar alles wollen, aber nicht alles haben. Wenn beispielsweise Art. 2 Abs. 2 Satz I GG als Abwehrrecht gegen den Staat konzipiert ist, dann wird das Thema Abtreibung grundrechtlich allenfalls relevant als an den Staat gerichtetes Verbot, die Schwangere zur Abtreibung zu zwingen. Die Pflicht des Staates, "werdendes Leben « mit den Sanktionen des Strafrechts gegen die Schwangere zu schützen, ist nur aus einer Grundrechtstheorie zu begründen, in der Art. 2 Abs. 2 Satz I GG als »Element objektiver Ordnung " gefaßt ist. Und nur wer Art. 3 Abs. 3 GG als demokratisches Teilhaberecht versteht, wird die Logik des »dissenting vote « des Richter Seufferts zum Radikalenjudikat überhaupt erst gedanklich nachvollziehen können, daß nämlich ein jeder Staatsbürger einen grundrechtlichen Anspruch darauf habe, »daß der Staat von seinen Organwaltern die Gewähr für die Beachtung dieses Grundrechtes fordert, so daß dieses Bürgerrecht insoweit auch für die Eignung zum öffentlichen Amt bestimmend ist, und zwar in weitem Vorrang vor den Rechten der Bewerber in bezug auf den Zugang zum öffentlichen Dienst « (BVerfGE 39, 375 f.). Wie gesagt, man kann das ja alles wollen, nur sollte dann Rechenschaft abgelegt werden über die grundrechtsdogmatischen Konsequenzen einer Grundrechtstheorie, die alles will.
Eine Verfassungstheorie, deren erstes Anliegen die rechtsstaatliche Freiheitssicherung ist, wird bei der Auslegung des Gleichheitssatzes zum Schwur gebracht, sieht sie sich doch einer mit den Namen Heller / F. Neumann / Abendroth verbundenen Theorietradition konfrontiert, die aus einem materialisierten Gleichheitssatz die sozialstaatliche Schubkraft des Rechtsstaates gewinnen will. Perels will beide verfassungstheoretischen Ansätze vereinigen, indem er für Art. 3 Abs. I GG den relativen Gleichheitsbegriff der herrschenden Verfassungsinterpretationen übernimmt und materialisiert in eine "Schutzfunktion für die Benachteiligten «, den Art. 3 Abs. 3 GG hingegen strikt formal im Sinne absoluter rechtlicher Gleichheit für alle Individuen auslegt. Das Dilemma, in das Perels' Grundrechtsinterpretation geraten muß, hat Schneider präzise prognostiziert. (Allerdings hat er es m. E. mit dem Prinzip der "Verallgemeinerungsfähigkeit aller Grundrechtsfunktionen « noch erheblich verschärft.) Die Normbereiche der beiden Gleichheitssätze sind nicht exakt trennbar und sagen deshalb wenig darüber aus, in welcher funktionalrechtlichen Beziehung die Grundrechte zu den Verfassungsstrukturen stehen. Perels versucht dem Einwand zu entgehen, indem er den Regelungsbereich des Art. 3 GG in zwei Materien zerschlägt. Im Bereich politischer Prozesse soll das rechtsstaatlich gefaßte Abwehrrecht des Art. 3 Abs. 3 GG Freiheit sichern, im gesellschaftlichen Bereich soll der sozialstaatlich aufgeladene Gleichheitssatz des Art. 3 Abs. I GG soziale Ohnmachtslagen ausgleichen. Das Beispiel, an dem Perels seinen Gedankengang exemplifiziert, benennt die Problematik des Ansatzes. Gehören Streik und Aussperrung zum gesellschaftlichen oder zum politischen Bereich? Wie und nach welchen Kriterien sind Gesellschaftlichkeit und Politik abzugrenzen und vor allem: wer entscheidet über die Zuordnung? Die Rechtsprechung zu Art. 16 Abs. 2 Satz 2 GG mag als warnendes Beispiel für die Schwierigkeiten dienen, in die die Juristerei gerät, wenn sie das »Politische « justiziabel machen muß. Aber selbst wenn die Differenzierung zunächst einmal akzeptiert wird, fällt es schwer, den Gewinn zu erkennen, den die »Berücksichtigung der sozialen Situation « gegenüber der Fassung des Gleichheitsbegriffs als Willkürverbot erbringt. Worin liegt der methodische Unterschied zwischen dem Satz "Gleiches darf nicht ungleich, Unglei- 
ches darf nicht gleich behandelt werden « und dem Appell, unterschiedliche Machtlagen nicht über einen Kamm zu scheren? Erbringt der Ansatz mehr als eine stärkere Gewichtung zugunsten "sozialer " Argumente innerhalb des Kanons materialer Gerechtigkeitskriterien? Um bei Perels Beispiel zu bleiben: ist die Gleichsetzung von Streik und Aussperrung im Verfassungsrecht einem formalen Gleichheitsbegriff geschuldet oder ist es nicht vielmehr so, daß das Paritätsdogma Resultat einer verfassungsillegitimen Übersetzung sozialer Wirklichkeit in das Verfassungsrecht ist? Wenn dem so sein sollte, wäre es dann nicht angemessener, aus Art. 9 Abs. I GG verfassungsrechtliche Argumente gegen die Gleichsetzung zu gewinnen anstatt das Set der "sachlichen « Differenzierungen zu erweitern? Generell scheint mir auch hier die Position Abendroths überzeugender zu sein. Das Anliegen, um das es Perels geht, ist weniger ein grundrechtstheoretisches denn ein verfassungs- und rechtspolitisches: der Grundrechtskatalog des Grundgesetzes ist zu ergänzen um soziale Grundrechte, die Aussperrung ist durch einfaches Gesetz zu verbieten.

Betrachtet man die weiteren Beiträge unter der grundrechtstheoretischen Fragestellung, dann fallen zwei Autoren durch eine klare Positionsangabe auf. Podlechs kurze Studie zum "Recht auf Privatheit « ist eine Demonstration der Leistungsfähigkeit des klassischen Eingriffs- und Schrankendenkens auch angesichts neuer gesellschaftlicher Problemlagen, zu deren Kennzeichnung das Stichwort von der Freiheitsbedrohung durch informationelle Fremdbestimmung ausreichen soll. Das klassisch-liberale Grundrechtsverständnis sichert in dem Recht auf "private, persönliche, irgendwelchen öffentlichen Zwecken nicht verpflichtete Freiheit « (S. 53) die freie Überzeugungsbildung und damit die Erhaltung der Lernfähigkeit der Gesellschaft. Es ist interessant festzustellen, daß Podlech, obwohl aus einer ganz anderen verfassungstheoretischen Tradition kommend, im Ergebnis mit Abendroth übereinstimmt. Die Sicherung politischer Demokratie basiert auf der Priorität eines radikal rechtsstaatlichen Grundrechtsschutzes.

Ähnlich konsequent ist Keßlers Aufsatz zur Koalitionsfreiheit. Die Gegenposition, zu der sich sein Ansatz negatorisch bestimmt, ist nicht die Konzeption sozialer oder politischer Teilhaberechte, sondern eine institutio-

\section{J.Schweitzer Verlag}

Wichtige Gesetze und Entscheidungen in Auszügen

$$
\begin{aligned}
& \text { Alexander Eberth } \\
& \text { Drogenrecht }
\end{aligned}
$$

Ein Ratgeber für die Praxis der Berater, Therapeuten und anderer Helfer in der Drogenarbeit. Von Alexander Eberth, Rechtsanwalt in München. DIN A 6. VIII, 112 Seiten. 1981. Kartoniert DM 9,80. Bei Sammelbestellungen ab 20 Expl. je DM 7,80 ISBN 388709 ool 2

Für Mitarbeiter in Drogenberatungsstellen und therapeutischen Einrichtungen, für Bewährungshelfer und andere Personen, die beruflich oder ehrenamtlich Dronenabhangigen ur rogenabhängigen und Drogengefährdeten helfen wollen.

Neben dem neuen Betäubungsmittelgesetz (ab 1.1.1982) sind in dem Buch die wichtigsten Gesetze in Auszügen zusammengestellt, soweit sie für die Arbeit von Bedeutung sind. Dies gilt sowohl für das Strafrecht, Bewährungsfragen, erziehungsrechtliche Probleme als auch für das Gebiet der Kostenerlangung. Berücksichtigt wurden auch die Probleme der Träger und Mitarbeiter selbst. Außerdem enthält das Buch einige wichtige Hinweise und Entscheidungen zu grundsätzlichen Fragen, u.a. des Zeugnisverweigerungsrechts, der vorläufigen Kostenerlangung und zum Umgang mit Zollschulden.

Das handliche Format ermöglicht dem Benutzer, auch außerhalb seines Arbeitsplatzes die rechtlichen Grundlagen für seine Arbeit parat zu haben.

In Vorbereitung für Winter 1981/82: Eberth/Müller, Das neue Betäubungsmittelgesetz. Kommentar und Anleitung für die Praxis. Von Alexander Eberth und Dr. Eckhart Muiller, Rechtsanwälte in München. Oktav Ca XVI, 160 Seiten. 1981. Kartoniert ca. DM 24, (Vorbe stellpreis bis 31.12 .1981 ; späterer Ladenpreis ca. DM 28,--)

ISBN 3887090039 
nelle Grundrechtsauffassung. Keßler dekliniert an den verfassungsrechtlichen Positionen zur paritätischen Mitbestimmung die These durch, daß die Fassung der Koalitionsfreiheit als institutioneller Garantie demokratieverkürzende Wirkungen zeitige, während das Verständnis von Art. 9 Abs. 3 GG als Freiheitsrecht von Individuen und Verbänden die Offenheit der Koalitionsgarantie gegenüber gesetzlichen Mitbestimmungsregeln erhalte. Weil die grundrechtstheoretische Problematik insoweit verschoben ist, als ein Freiheitsrecht gerade nicht zur Abwehr von Interventionen des Gesetzgebers bemüht wird, liefern die Ausführungen zum Verhältnis von Grundrechtstheorie und -dogmatik einen Beleg, daß rechtsstaatliche Formtypik und politische Demokratie nicht notwendig in einen Gegensatz treten müssen. Denn ein liberales Grundrechtsverständnis beschränkt nicht nur den demokratischen Gesetzgeber, indem es Grundrechte "demokratiefest « macht, sondern es beläßt infolge seiner strengen Eingrenzung grundrechtlicher Regelungsbereiche gesetzgeberischen Initiativen einen breiten und vor allem berechenbaren Entfaltungsspielraum.

Hartmann leitet seine Beiträge zu Art.5 Abs. I und 3 GG mit einer Philippika gegen die nicht zuletzt von der Rechtsprechung des BVerfGs mitbegründete "Über-Verfassung" ein, die mit dem geltenden Verfassungsrecht auch Grundrechte partiell außer Kraft setzt und die »Inversion der Verfassung von Freiheitsverbürgung $\mathrm{zu}$ Staatserhaltung und Affirmation praktiziert«. (S. 96). Die Kritik impliziert die Konzeption von Meinungsund Wissenschaftsfreiheit als Abwehrrechte. Bemerkenswert ist dabei ein eigentümliches Oszillieren zwischen einer liberalen Grundrechtstheorie und einem demokratisch-funktionalistischen Ansatz. "Des Schutzes durch das Recht der Meinungsfreiheit . . . bedürfen nur solche Meinungen und Menschen, . . . die sich, im umfassendsten Sinne, in Opposition befinden «. (S. I I० f.).

Eine analoge Gedankenführung findet sich in Seiferts Ausführungen zur Vereinigungsfreiheit. In der Sache ist seine Interpretation der mindestens gleichen rechtsstaatlichen Radikalität verpflichtet. So wird der Verbotsvorbehalt des Art. 9 Abs. 2 GG strikt positivistisch (»... sind verboten «.) ausgelegt, um jenen Zwischenzustand der Halblegalität auszuschalten, in dem die »hoheitlichen Verrufserklärungen « insbesondere der Verfassungs- schutzberichte im Namen eines nur scheinbar liberalen Verhältnismäßigkeitsprinzips die Vereinsfreiheit aushöhlen. Dann aber wird die Vereinigungsfreiheit zum „Oppositions-Grundrecht « überhöht und funktionalisiert "für diejenigen, die darauf hinwirken, daß etwas anders wird «. (S. 175).

Eigentümlich ist an Hartmanns und Seiferts Ausführungen der Tatbestand, daß ihre flüchtigen Bezugnahmen auf eine demokratische Grundrechtstheorie keinerlei Einfluß auf die konkrete Arbeit der Grundrechtsinterpretation ausüben. Die demokratietheoretischen Reflexionen sind bloßes Begründungsornament. Der Leser gerät in Versuchung, Spekulationen anzustellen, ob hier nicht ein linkes Vorverständnis in dem Sinne Wirkung zeitigt, daß von rechtsstaatlicher Freiheitssicherung nur dann gesprochen werden darf, wenn die hehre demokratische Funktion des grundrechtlichen Freiheitsschutzes argumentativ nachgeschoben wird.

Mehr als ein Begründungsornament ist das demokratische Grundrechtsverständnis in Otts Aufsatz zur Versammlungs- und Demonstrationsfreiheit. Ott streitet gegen die individualrechtliche Auslegung der Versammlungsfreiheit, sie verkümmere dann leicht zur Belanglosigkeit, und insistiert mit Hesse darauf, daß sie Konkretisierung des demokratischen Prinzips sei und zur Verwirklichung der Volkssouveränität beitrage. Der Einwand gegen demokratische Funktionalisierungen des Grundrechtsschutzes ist im Beitrag Podlechs nachzulesen. Weil eine freiheitliche Demokratie auf den Zwang zum Politischen verzichten muß, gehört zur Freiheit zuvörderst die Freiheit, unpolitisch sein zu dürfen. Otts These, Freiheit erweise sich erst im Schutze der Freiheit politischer Opposition, ist zu glatt, als daß sie nicht Widerspruch hervorzurufen vermöchte. Um die Freiheit politischer Oppositionsgruppen braucht uns weniger bange zu sein als um die Freiheit jener, die aus der Politik aussteigen, denen Demokratie und Volkssouveränität schnurzegal sind. Im Schutze der Freiheit beispielsweise des Antietatismus der Zeugen Jehovas oder der Radikalität alternativer Lebensformen, die den politischen Geschäften von Mehrheit und Minderheit den Rükken kehren, zeigt sich die Freiheitsfähigkeit einer Gesellschaft. Eine demokratisch-funktionalistische Grundrechtstheorie tut sich schwer, solchen unpolitischen Gruppen Frei- 
heit zu gewähren, weil der Grad der Freiheitsgewährung bemessen wird nach der politischen Qualität des Freiheitsgebrauchs. Die zwei klassischen Entscheidungen des BVerfGs zur Meinungsfreiheit, das "Lüth-Urteil« und das "Hausgemeinschafts-Urteil «, sind die dogmatischen Konsequenzen einer Grundrechtstheorie, die den Grundrechtsschutz an demokratische Grundrechtsgebrauchsqualitäten rückbindet.

Nicht allein die grundrechtstheoretische Fragestellung, die der Rezension als Leitfaden diente und auf die sie sich bewußt beschränkte, macht Perels Sammelband lesenswert. Erwähnt seien noch die Grundrechtsgeschichte, die Schneider und - speziell zu Art. 3 GG - Perels geschrieben haben, sowie die von allen Autoren geleistete rechtsdogmatische Arbeit. Auch für diese und weitere Aspekte des Buches gilt das Urteil, daß die Beiträge den fachjuristischen Vergleich nicht zu scheuen brauchen.

Volker Neumann

Diemut Majer, "Fremdvölkische im Dritten Reich. Ein Beitrag zur nationalsozialistischen Rechtssetzung und Rechtspraxis in Verwaltung und Justiz unter besonderer Berücksichtigung der eingegliederten Ostgebiete und des Generalgouvernements. Schriften des Bundesarchivs, Band 28. Harald Boldt Verlag, Boppard I98I, I050 Seiten, 22 Abbildungen, I Karte, Leinen, DM 96,-

Die juristische Habilitationsschrift von D. Majer behandelt mit einer Fülle von Material in verdienstvoller Weise ein von der allgemeinen Geschichtsschreibung bisher noch nicht bearbeitetes wichtiges Gebiet der deutschen Zeitgeschichte - die Pervertierung des Rechtsstaats zwischen 1933 und 1945, in seiner besonders brutalen Konsequenz am deutlichsten darzustellen am Ausnahme- und Sonderrecht für Fremdvölkische im Dritten Reich«. Gemeint sind vor allem Juden und Polen im deutschen Machtbereich. In einer breiten Einleitung skizziert die Vfn. zunächst als Hintergrund die besondere Struktur von Recht und Verwaltung im NS-Staat, einschließlich »Führerprinzip« und »Vorherrschaft der Partei über den Staat «, weiter zugespitzt auf das eigentliche Thema: "Das
Uwe Wesel 457

\section{Uwe Wesel} Aufklärungen über Recht

Zehn Beiträge zur

Entmytholisierung

suhrkamp taschenbuch

wissenschaft 368. DM 10,-

Das Demokratiegebot des

Grundgesetzes ist am

schwächsten verwirklicht im Bereich der dritten Gewalt. Im Gegensatz zum Parlament und zur Regierung entzieht sich die Justiz weitgehend einer öffentlichen Kontrolle,weil ihre Sprache, Begrifflichkeit und die Kompliziertheit ihrer logischsystematischen Argumentation hohe Barrieren aufrichtet für eine Nachprüfung durch Außenstehende. Tagtäglich ergehen im Namen des Volkes unzählige juristische Entscheidungen, die das Leben der Gesellschaft genauso beeinflussen und steuern wie die Tätigkeit von Parlament und Regierung. Einsichtig sind sie selten. Eine Kritik von außen ist kaum möglich. An diesen Problemen setzen Uwe Wesels Beiträge ein. In allen Buchhandlungen.

Suhrkamp Taschenbuch Verlag 
Prinzip der 'völkischen Ungleichheit< (Sonderrecht)«. Als »Objekte der Verwirklichung der >völkischen Ungleichheit « nahm der NS-Staat Juden und "andere sfremdvölkische Minderheiten « ins Visier. "Das Prinzip des Sonderrechts « erweist sich "als Zentralbegriff " zur Charakterisierung der NS-Politik gegen Juden vor und im 2. Weltkrieg, gegen die Polen im 2. Weltkrieg.

In zwei großen Teilen, jeder noch einmal in sich reich untergliedert, rückt die Vfn. schon mit den Teilüberschriften daher »das Prinzip des Sonderrechts « ins Zentrum der Analyse, jeweils "auf dem Gebiet des öffentlichen Rechts « und "im Bereich der Justiz «. Drei Abschnitte im Ersten Teil behandeln »die Verwirklichung der >völkischen Ungleichheit«", jeweils im Altreich, in den »eingegliederten Ostgebieten «, d. h. vom Großdeutschen Reich 1939 annektierten Gebiete Polens, schließlich im Generalgouvernement Polen. Entsprechend den territorialen Bedingungen im Altreich, in den annektierten polnischen Gebieten und im Generalgouvernement ist auch der Zweite Teil untergliedert, der die Konsequenzen des Sonderrechts für Strafrecht und Bürgerliches Recht behandelt.

Insgesamt erweist sich die Einführung des Sonderrechts gegen Juden im Altreich seit 1933, später auch im Großdeutschen Reich vor und im 2. Weltkrieg zur Diskriminierung, Verdrängung und Vernichtung der Juden durch eine Fülle eskalierender Verordnungen, Gesetze, Maßnahmen und Gerichtsurteile im neuen "völkischen" Sinne als beklemmender Präzedenzfall und zugleich als Übungsfeld für die NS-Machtpolitik nach der Eroberung Polens im September 1939: Das im Innern gegen die Juden bereits »erprobte « Prinzip des Sonderrechts wurde nun nach Außen auf die unterworfenen Polen ausgedehnt. Obwohl sich die NS-Machthaber ängstlich bemühten, ihre Schritte in juristische Formen zu gießen, produzierten sie damit nur blanken Terror. Selbstredend verstieß die NS-Politik gegen eine wesentliche Basis des Völkerrechts, die Haager Landkriegsordnung: Schon die Annexion der »eingegliederten Ostgebiete « ins Großdeutsche Reich noch mitten im Krieg verletzte die Haager Landkriegsordnung. Auch das Generalgouvernement Polen als "angegliedertes Gebiet« und »Muster der zukünftigen deutschen Kolonien « wurde abwechselnd, wie es die Opportunität juristischer Konstruktionen gerade erforderte, rechtlich jeweils als Inland oder Ausland behandelt, wenn sich nur für neue Diskriminierungs- und Repressionsmaßnahmen gegen Juden und Polen eine technisch-juristische Verbrämung fand.

In den annektierten Gebieten schälten sich drei Stufen der Verwirklichung der svölkischen Ungleichheit«« heraus - »sinngemäße Anwendung deutschen Rechts" (bis Mai I940), Sonderrecht (bis Herbst 194I), "Schaffung offenen Sonderrechts für Nichtdeutsche«" (seit Herbst 194I). Im Generalgouvernement ergab sich eine Komplikation aus dem Fortbestehen des polnischen Justizwesens, dem ein deutsches über- und vorgeordnet wurde. Entsprechend dem zentralen Prinzip des Sonderrechts wird die große Bedeutung (deutscher) Sondergerichte deutlich. In ihrer trockenen (manchmal unnötig schleppenden) und nüchternen Juristensprache liefert die Arbeit so eine aus den Quellen erarbeitete Illustration für die totale Regulierung aller Lebensbereiche durch den NS-Staat, in Umkehrung aller bisherigen Rechtsgrundsätze: Alles war im Prinzip dem Interesse des nationalsozialistischen Staatsapparats unterzuordnen, alles wurde im Prinzip mit vagen Generalklauseln verboten, so daß sich der legale Bewegungsraum für das Individuum nur aus Ausnahmeregeln ergab. So entstand eine totale Rechtsunsicherheit, verschärft durch das Kompetenzgewirr sich überschneidender ordentlicher Gerichtsbarkeit und vielfältiger Sondergerichte. In aller Schärfe durchgeführt wurde dieses totalitäre Prinzip der Rechtsperversion in Deutschland gegen die Juden, anschließend übertragen auf das als fast völliges Rechtsvakuum behandelte besiegte Polen, besonders im Generalgouvernement.

Das Gesamtergebnis war eine in der Nüchternheit der juristischen Kategorien gehaltene geradezu beklemmende Fundierung deutscher Macht- und Vernichtungspolitik gegenüber Juden und Polen. In ihrer Rekonstruktion der juristischen Seite deutscher Unterdrückungspolitik gegen jüdische Reichsbürger und polnische Ausländer liefert die Arbeit zugleich einen wertvollen Beitrag zur historisch-politischen Aufarbeitung und Klärung unseres Verhältnisses zu Polen und Juden. Es ist geradezu ein wissenschaftliches Gegenstück zu der Primärquelle, die gleichsam die praktische politische Ergänzung bietet, zum Diensttagebuch von Generalgouverneur Hans Frank, das in seiner erdrückenden (und 
deshalb so schwer zu bewältigenden) Fülle seit einigen Jahren vorliegt.

Für die allgemeine Geschichtsforschung und die jüngere deutsche Rechtsgeschichte stellt die Arbeit zweifellos eine bedeutende Leistung dar, die weit über Juristen hinaus ihre Wirkung haben wird, wozu auch - trotz des enormen Umfanges, der gelegentlichen
Schwerfälligkeit des Juristenstils und des stolzen Preises - die Aufnahme in die Schriftenreihe des Bundesarchivs hoffentlich beitragen wird. Um so mehr ist es zu bedauern, daß die Arbeit vom Fachbereich Jura der FU Berlin als Habilitationsschrift abgelehnt wurde, u. a., weil die NS-Zeit »mit dem heutigen Recht nichts zu tun hat«.

Imanuel Geiss 